\title{
Caracterização da mineração e do potencial mineral de óxido de ferro e argila do município de Caicó-RN a partir de técnicas de sensoriamento remoto
}

\author{
Characterization of the mineral and mineral potential of iron oxide and clay of the \\ municipality of Caicó-RN from remote sensing techniques
}

\author{
SILVA $^{1}$, L. C. M.; VITAL ${ }^{2}$, S. R. O.; BATISTA ${ }^{3}$, C. T. \\ luanamariz21@hotmail.com;
}

\begin{abstract}
Resumo
Os recursos naturais são de fundamental importância para vida humana, dentre eles, os recursos minerais têm grande utilidade econômica e aplicações diversas. A presente pesquisa tem como objetivo caracterizar a mineração e identificar o potencial mineral do município de Caicó (RN), através do mapeamento das áreas mais propícias à concentração de determinadas substâncias, utilizando dados de processos minerários pré-existentes, relacionando-os com dados obtidos a partir de sensores orbitais. Para isso, foram utilizadas imagens do TM/LANDSAT, aplicando a técnica de operação razão de bandas, tendo como resultado o realce das substâncias de interesse. Logo, foi possível identificar a ocorrência de óxido de ferro e argila e relacioná-las com a geologia local. A comparação entre as unidades geológicas e as substâncias identificadas mostra bastante coerência dos resultados.
\end{abstract}

Palavras-chave: Potencial Mineral; Sensoriamento Remoto; Município de Caicó-RN.

\begin{abstract}
Natural resources are of fundamental importance for human life, among them, mineral resources have great economic utility and diverse applications. The present research aims to characterize mining and identify the mineral potential of the municipality of Caicó (RN), by mapping the areas most conducive to the concentration of certain substances, using data from pre-existing mining processes, relating them with data obtained from orbital sensors. For this, images of the TM/LANDSAT were used, applying the technique of operation of band ratio, resulting in the enhancement of the substances of interest. Therefore, it was possible to identify the occurrence of iron oxide and clay and to relate them to the local geology. The comparison between the geological units and the identified substances shows a very consistent results.
\end{abstract}

Keywords: Mineral Potential; Remote Sensing; Municipality of Caicó-RN.

\section{INTRODUÇÃO}

Desde o início da humanidade os recursos naturais têm ligação direta com o desenvolvimento das civilizações e com o surgimento de novas técnicas, sendo hoje, indispensáveis na vida moderna para o crescimento econômico e a evolução social. Os mesmos se distinguem em recursos renováveis e não renováveis.

\footnotetext{
${ }^{1}$ Luana Carla Mariz da Silva, Departamento de Geografia, Universidade Federal do Rio Grande do Norte, Caicó-RN, Brasil.

${ }^{2}$ Saulo Roberto de Oliveira Vital, Departamento de Geografia, Universidade Federal do Rio Grande do Norte, Caicó-RN, Brasil.

${ }^{3}$ Clístenes Teixeira Batista, Geógrafo, Consultor Ambiental, Fortaleza-CE, Brasil
} 
Os recursos renováveis podem ser classificados como aqueles que têm maior capacidade de reposição numa escala de tempo relacionada ao consumo humano, diferente dos recursos não renováveis, que levam milhões de anos para se formarem e serem utilizados novamente (LEPSCH, 2011). Nesse caso, dentre os não renováveis estão, em sua maioria, os recursos minerais.

O Estado do Rio Grande do Norte é caracterizado por uma geologia que engloba desde rochas ígneas e metamórficas arqueanas e proterozóicas (pré-cambriano) até importantes unidades fanerozóicas sedimentares, coma a Bacia Potiguar, além de depósitos Cenozóicos, como a Formação Barreiras, que constitui uma cobertura de plataforma, capeadora de outras bacias marginais; e, por fim, os depósitos eólicos, coluvionares e aluvionares do Quaternário (ANGELIM et al. 2006b).

O referido estado possui destaque por ter grande potencial mineral, com uma produção bastante diversificada de granito, calcário, scheelita, ferro, argila, além de ser um grande produtor de petróleo, gás natural e sal.

Esse potencial mineral e a diversidade de recursos estão associados às características geológicas da região, que propicia a ocorrência de recursos minerais abundantes, com aplicações para diversos fins (LIMA et al. 2012).

A área de estudo faz parte deste domínio, mais precisamente no complexo Caicó, que tem como característica principal, uma grande diversidade de minerais provenientes de rochas metamórficas (ANGELIM et al. 2006a).

Segundo Fitz (2008, p. 109) o sensoriamento é uma "técnica que utiliza sensores para a captação e registro à distancia, sem o contato direto, da energia refletida ou absorvida pela superfície terrestre". Esta técnica é uma ferramenta de conhecimento do espaço geográfico, sendo muito aplicado em trabalhos científicos, planejamentos governamentais, previsões meteorológicas e outras diversas aplicações.

A técnica de Processamento Digital de Imagens de sensoriamento remote fornece e permite a identificação de informações para estudos geológicos e pesquisas de substancias minerais que tem bastante relevancia economica. Em função disso há um ganho à exploração geológica em termos de economia de recursos vinculados à prospecção mineral (FOLLADOR, 2004).

Diante disso e da diversidade de substâncias identificadas pelo DNPM (Departamento Nacional de Produção Mineral), a pesquisa tem como objetivo, relacionar os dados e 
informações já existentes do DNPM com os dados obtidos a partir de técnicas de sensoriamento remoto de duas substâncias de grande importância econômica, óxido de ferro e argila e relacioná-las com o contexto geológico local.

\section{METODOLOGIA}

Para a execução da presente pesquisa, foram utilizadas informações bibliográficas acerca do assunto e bases cartográficas diversas. Em relação aos dados cartográficos, no primeiro momento, foi realizada uma análise dos dados fornecidos pelo DNPM, referentes aos atuais processos minerários (todo tipo de processo feito no DNPM, como o requerimento, alvará de pesquisa, registro de licenciamento até as últimas etapas do processo) da área de estudo, além de informações obtidas no Mapa Geológico do Rio Grande do Norte, fazendo relação da sua geologia e as substâncias detectadas a partir das técnicas de PDI (Processamento Digital de Imagens).

Para a elaboração dos mapas de localização, geologia e substâncias minerais, foram utilizadas informações a partir de fontes de dados, tais como: IBGE (Instituto Brasileiro de Geografia e Estatística), DNPM (Departamento Nacional de Produção Mineral) CPRM, (Serviço Geológico do Brasil); processados em um SIG (Sistema de Informações Geográficas) no software ArcGIS 10.3.

Para os mapas do potencial mineral, foram utilizadas imagens do satélite TM/LANDSAT, órbita 215, pontos 64 e 65, de 23 de setembro de 2008, com resolução espacial de 30 metros e resolução radiométrica de 8 bits, adquiridas no site Catálogo de Imagens do Instituto Nacional de Pesquisas Espaciais (INPE). Em seguida, confeccionou-se o mosaico a partir das imagens 64 e 65 referentes às bandas 1, 3, 5 e 7.

Posteriormente, as bandas mencionadas foram georreferenciadas, utilizando uma imagem do OLI/LANDSAT como base, uma vez que as mesmas são distribuídas com certo nível de pré-processamento (registro e correção atmosférica). Na razão de bandas de argila representado pelo o infravermelho de ondas curtas, corresponde a (SWIR) 1: 1, 55-1, $75 \mu \mathrm{m}$, (SWIR2) 2: 2,08-2,35 $\mu \mathrm{m}$ refere-se as bandas 5 (SWIR1) e 7 (SWIR2), onde foi aplicado a Equação 1, processada no ArcGIS 10.3 . Este índiece funciona com qualquer sensor multiespectral com bandas que se enquadram nas faixas listadas.

$$
\text { Clay }=(\text { SWIR1/SWIR2) }(1)
$$


Já a razão de óxido de ferro revela rochas hidrotermicamente alteradas que foram submetidas à oxidação de sulfetos que possuem ferro. Onde o vermelho corresponde a 0,630,69 $\mu \mathrm{m}$ e o azul a 0,45-0,52 $\mu \mathrm{m}$, o que são equivalente as bandas 3 (vermelho) e 1 (azul), na qual foi aplicado a equação a baixo no ArcGIS 10.3 . De posse dos dados, foram comparados os resultados obtidos na imagem e com os dados dos processos minerários obtidos junto ao DNPM e da geologia do local.

$$
\text { Iron Oxide }=(\text { Red/Blue })(2)
$$

\subsection{Caracterização da Área Estudada}

O município de Caicó encontra-se localizado na microrregião do Seridó Ocidental do Rio Grande do Norte, distando cerca de $256 \mathrm{~km}$ da capital do estado (Figura 1). O referido município abrange uma área de $1.228,583 \mathrm{~km}^{2}$, com uma densidade demográfica de 51,04 habitantes por $\mathrm{km}^{2}$, e uma população estimada de 67.747 habitantes (IBGE, 2016). A cidade faz limite com os municípios de Jucurutu, Florânia, Cruzeta, São José do Seridó, Jardim do Seridó, Ouro Branco, São João do Sabugi, Serra Negra do Norte, Timbaúba dos Batistas e São Fernando (CPRM 2005).

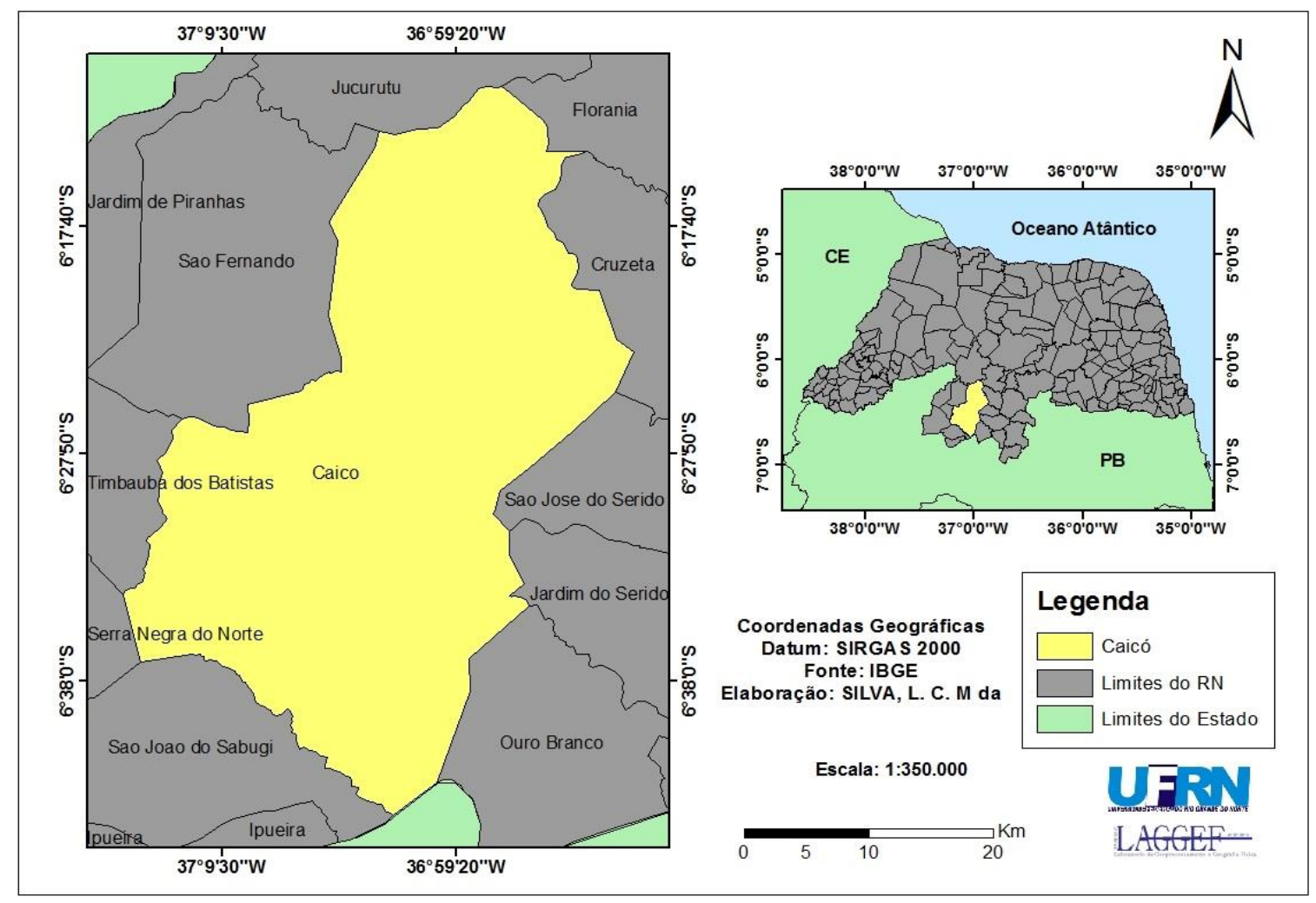

Figura 1. Mapa de localização do município de Caicó-RN. Fonte: Elaboração dos autores. 


\section{RESULTADOS E DISCUSSÃO}

\subsection{Quadro Mineral de Caicó a Partir de Dados do DNPM}

Para se obter um panorama geral do quadro mineral de Caicó, foram utilizados dados do SIGMINE (Sistema de Informações Geográficas da Mineração) do DNPM, que fornece registros de alvarás ou processos minerários. Nesse caso, os dados não corresponderam necessariamente às atividades de mineração em curso no município, uma vez que pode haver desatualização ou informações imprecisas sobre a substância e o uso das mesmas.

A existência de processos minerários de uma variada gama de substâncias minerais indica a diversidade mineral no município analisado, com cerca de 112 processos minerários no dia 29 de fevereiro de 2016, dentre eles: concessão de lavra, licenciamento, autorização de pesquisa, requerimento de pesquisa, dentre outros, com 15 tipos de substâncias distintas, tais como: areia, argila, bismuto, calcário, fosfato, gabro, granito, berílio, cobre, ferro, ouro, tungstênio, mármore, quartzito e scheelita (Figura 2).

Apesar de todo esse potencial, vale ressaltar que nem toda ocorrência mineral significa que seja viável sua exploração, caso a mesma não ocorra de forma suficientemente concentrada para viabilizar sua exploração ou caso os custos de extração do minério superem seu valor de mercado.

Segundo Pouchain (2011), muitos dos processos minerários dirigidos ao DNPM são em regime de autorização de pesquisa, que consiste numa modalidade em que é necessário realizar uma pesquisa geológica e/ou geofísica em um prazo de 1 a 3 anos, para se determinar o aproveitamento mineral e se avaliar a viabilidade técnica e econômica da exploração antes da concessão de lavra. Com isso, é possível que nem todo processo minerário se configure em uma mina economicamente viável pelos motivos expostos acima.

A partir da observação dos processos minerários, o número maior de solicitações é de minério de ferro e ouro, este último tendo sua origem de veios hidrotermais e metaconglomerados. Os veios são compostos principalmente de quartzo, de pequeno porte, sendo que, sua formação está associada a zonas de cisalhamento que existem na região (ANGELIM et al. 2006a).

Com base no conhecimento preliminar da geologia local, presume-se que deve haver alguma superestimação na ocorrência dessas substâncias, que estão alojadas, em maior parte, em ortognaisses do Complexo Caicó, cujo potencial para ocorrência de ouro, e principalmente 
de ferro, não condiz com o alto interesse dos agentes da mineração, tendo em vista o grande número de processos minerários.

Além do ferro e do ouro, os minérios de cobre, granito e quartzito tem bastante ocorrência entre os processos minerários. Por outro lado, nota-se a pouca presença de processos de extração de argila e areia, substâncias indispensáveis à construção civil. Mas, isso, provavelmente, se deve ao regime fluvial das principais drenagens que ocorrem no município, onde as baixas vazões não permitem acumulação de sedimentos em volume considerável.

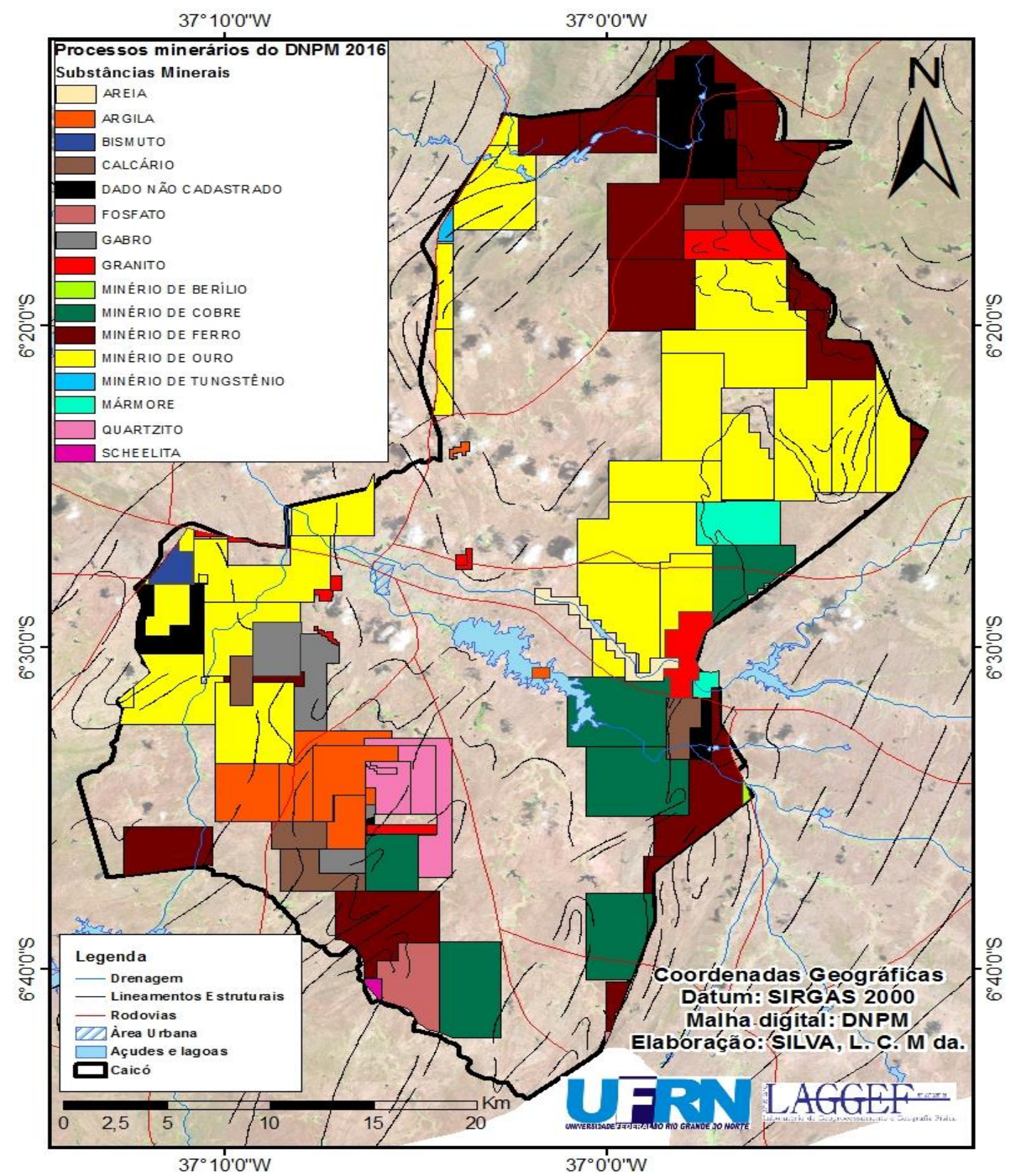

Figura 2. Mapa de processo minerário de substâncias minerais do município de Caicó/RN. Fonte: Elaboração dos autores. 


\subsection{Levantamento do Potencial Mineral Através de PDI.}

\subsubsection{Detecção de Óxido de Ferro}

O Processamento Digital de Imagens de sensores remotos permite um melhor aspecto visual das estruturas físicas da superfície da Terra.

As razões de bandas utilizadas foram sugeridas por Drury (1987) a qual foi utilizada como referência para está pesquisa. As razões de bandas 3/1 (óxido de ferro) e 5/7 (argila), respectivamente, foram empregadas por apresentar resultados significativos, conforme foi demonstrado e citados em estudos anteriores como de Menezes et al 2006 e Crósta 1992.

A figura 3 expõe os resultados da aplicação da razão 3/1, empregada na detecção de óxido de ferro. As manchas em vermelho indicam a presença desta substância e os processos minerários destacados são aqueles relacionados aos minérios de ferro solicitados ao DNPM.

Com isso, pode-se identificar que, em grande parte dessas solicitações, foi possível detectar óxido de ferro, havendo uma consonância entre os dados obtidos por sensoriamento remoto e as substâncias requeridas.

A ocorrência desse material está associada à geologia local, nas unidades litoestratigráficas do Complexo Caicó, de origem metavulcanossedimentar, na Formação Serra dos Quintos e Formação Jucurutu. Esses depósitos ferríferos estão associados à ocorrência de magnetita, hematita e quartzo, podendo, ainda, conter martita e limonita (FERREIRA \& SANTOS, 2000).

O minério de ferro é uma das substâncias mais viáveis à exploração, e pode ser classificado quanto à sua composição, como: óxido, carbonato, sulfeto e silicatos, sendo o óxido de ferro, aquele de maior expressividade econômica (CARVALHO et al. 2013).

A detecção presente na Figura 3, tanto pode estar associada às formações ferríferas, como a outros tipos de formações geológicas, já que óxido de ferro pode estar presente em diversos minerais.

No minério de ferro, os óxidos de ferro possuem grande concentração, podendo ser, de fato, a única fase mineral presente. Nesse sentido, a Figura 3 mostra a associação do óxido de ferro com as formações geológicas, no intuito de estabelecer relações entre as informações. 


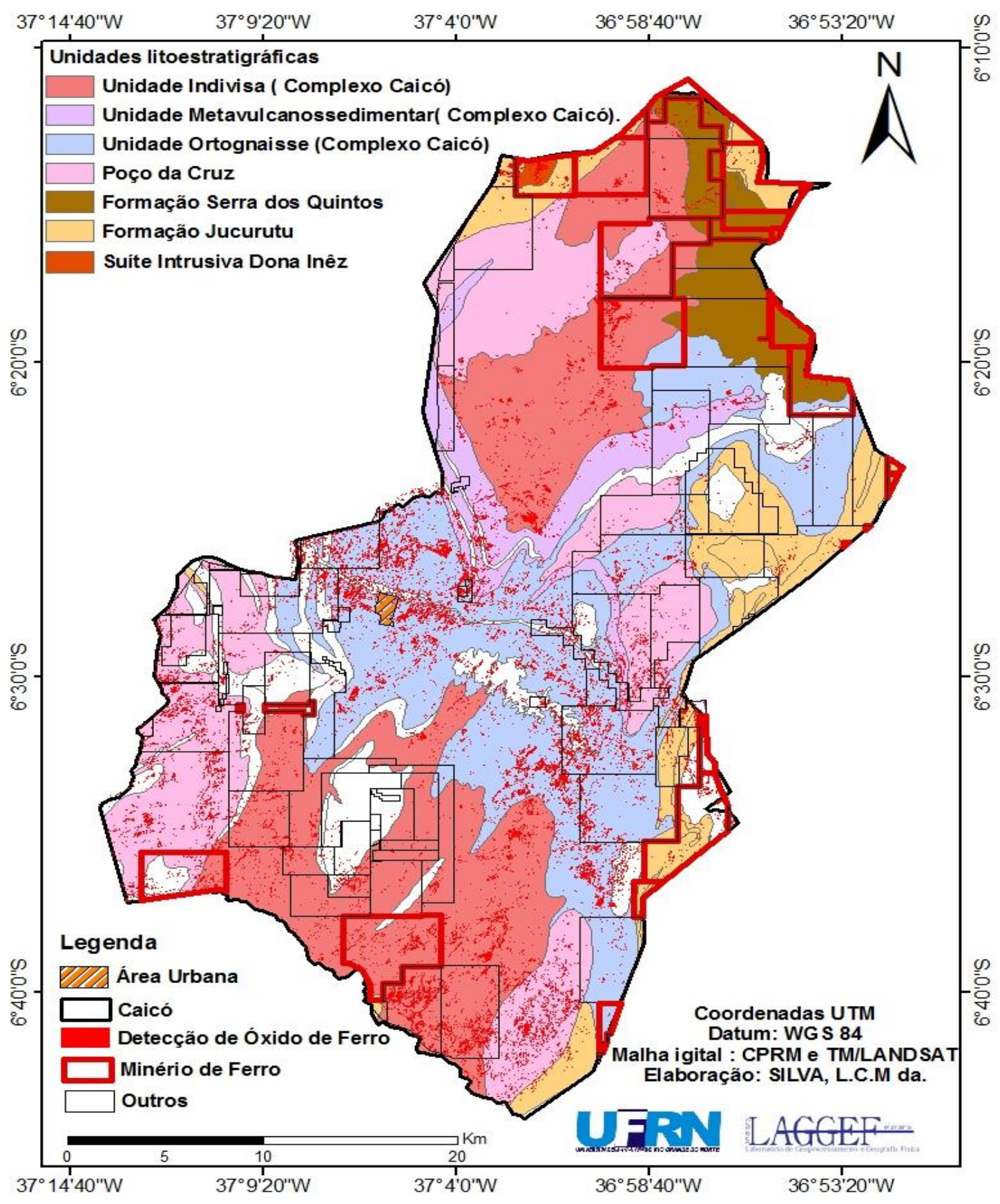

Figura 3. Mapa das unidades litoestratigráficas e detecção de óxido de ferro no município de Caicó/RN. Fonte: Elaboração dos autores

Desse modo, pode-se inferir que as formações ferríferas existentes no mapa geológico coincidem, em grande parte, com as possíveis ocorrências de óxido de ferro detectadas nas razões de bandas. As formações com maior presença de óxido de ferro estão presentes no Complexo Caicó, nas seguintes unidades: Metavulcanossedimentar, Ortognaisses, Indivisa e Poço da Cruz e a Suíte Intrusiva Dona Inez. Esta identificação do óxido nas várzeas está relacionada com o processo de intemperismo químico, onde acontece a oxidação. 


\subsubsection{Detecção de Argila}

Para a detecção de argila, foi adotado o mesmo procedimento, utilizando a razão de bandas 5/7, conforme exposto na Figura 4, os pontos em vermelhos foram realçados através da razão, sendo reconhecidos como argila. Os processos minerários do DNPM, por sua vez, estão destacados em azul, o que demonstra que grande parte da detecção de argila está dentro ou próximo dos processos, mesmo sendo poucos.

Segundo Angelim (2006a), esse tipo de argila pode ser classificado como várzeas, que estão relacionados a aluviões e planícies de inundações de rios. Com isso, foi possível identificar a relação das argilas com diversos fatores já citados, porém, o mais significante seria a presença do mesmo no entorno dos corpos hídricos.

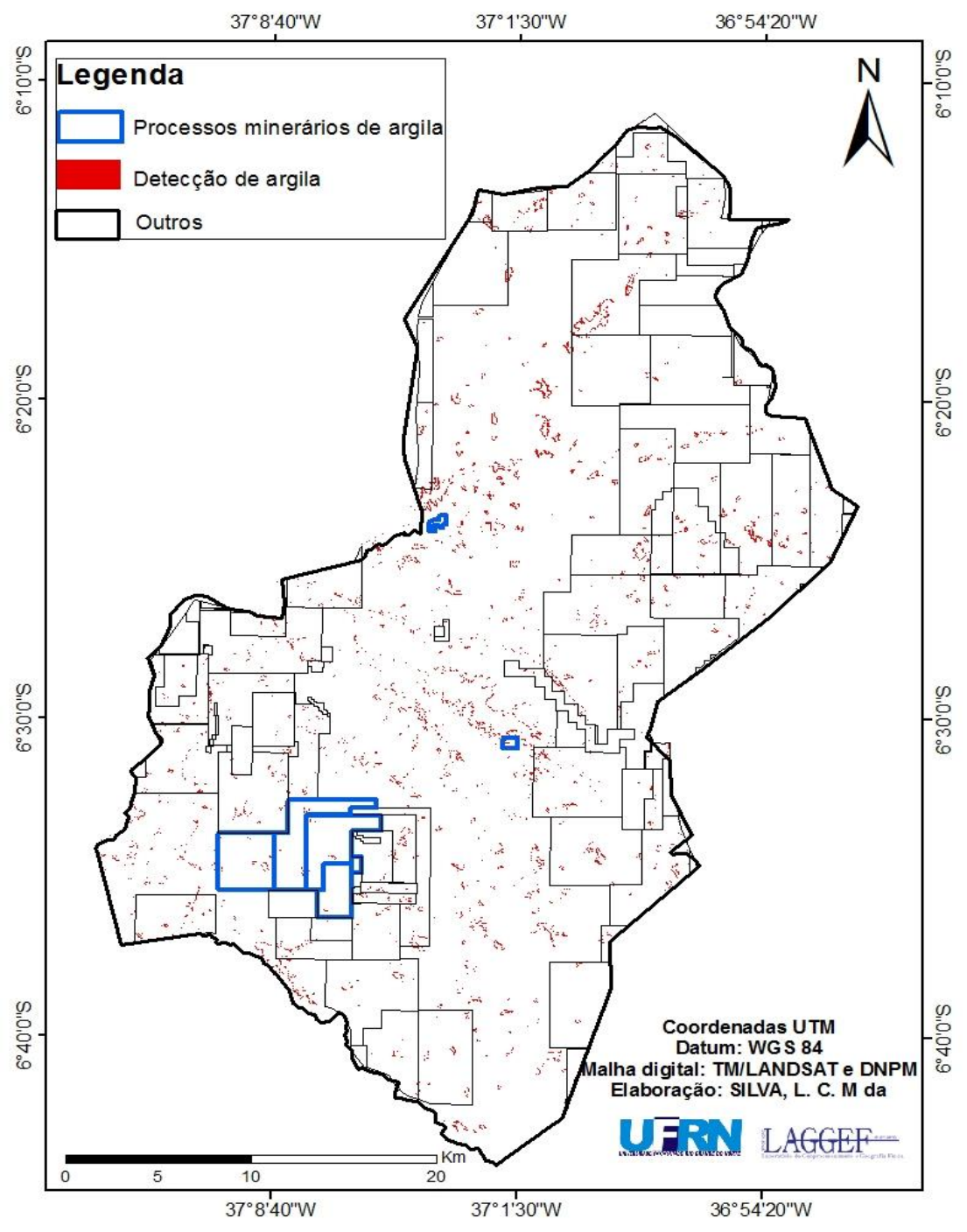

Figura 4. Mapa de detecção de argila no município de Caicó. Fonte: Elaboração dos autores. 


\section{CONSIDERAÇÕES FINAIS}

Apesar das limitações do sensor TM, sobretudo em relação à sua resolução espacial, o resultado foi satisfatório, pois identificou-se a ocorrência das substâncias analisadas através das técnicas de PDI, havendo consonância com os processos minerários do DNPM.

Logo foi possível constatar novas áreas ainda não diagnosticadas, o que, provavelmente, esteja relacionado a outros minerais do grupo dos óxidos, que estejam presentes em vários tipos de formações geológicas. Portanto, faz-se necessária a realização de outros estudos com a aplicação dessa técnica, para comprovar se há ocorrência de outras substâncias, podendo levar a descoberta de possíveis depósitos minerais.

Conclui-se, desse modo, que a pesquisa atingiu seu objetivo, que foi o de identificar e mostrar as possíveis ocorrências de óxido de ferro e argila com o uso das técnicas de sensoriamento remoto.

\section{REFERÊNCIAS}

ANGELIM, L. A. A.; NESI, J. R.; TORRES, H. H. F.; MEDEIROS, V. C.; SANTOS, C.A.; JUNIOR, J. P. V. \& MENDES, V. A. 2006a. Geologia e Recursos Minerais do Estado do Rio Grande do Norte: Texto Explicativo dos Mapas Geológicos e Recursos Minerais do Estado do Rio Grande do Norte. CPRM/SEDEC-RN/FAPERN. Recife, 119 p.

ANGEliM, L. A. A.; NESI, J. R.; TORRES, H. H. F.; MEDEIROS, V. C.; SANTOS, C.A.; JUNIOR, J. P. V. \& MENDES, V. 2006b. Projeto Mapa Geológico e de Recursos Minerais do Estado do Rio Grande do Norte. Mapa Geológico do Rio Grande do Norte. Recife: CPRM/FAPERN. Escala 1:500.000.

CARVAlHO, P. S. L.; SIlVA, M. M DA.; ROCIO, M. A. R. \& MOSZKOWICZ, J. 2013. Minério de Ferro. BNDS.

CRÓSTA, A.P. Processamento Digital de Imagens de Sensoriamento Remoto. Instituto de Geociências, UNICAMP, Campinas, SP. 1992.

DRURY, S.A. Image Interpretation in Geology. London: Allen\& Unwin, 1987, p. 243. 
FERREIRA, C. A. \& SANTOS, E. J. 2000. Programa Levantamento Geológico Básico do Brasil. Jaguaribe SE. Folha SB. 24-z. Estados do Ceará, Paraíba, Pernambuco Rio Grande do Norte. CPRM, Recife.

FOLLADOR, M. A caracterização mineral pelo sensoriamento remoto. VII Curso de Especialização em Geoprocessamento. Belo Horizonte, 2004.

FITZ, P. R. Cartografia Básica. São Paulo: Oficina de Textos, 2008.

Instituto Brasileiro de Geografia e Estatística (IBGE). Cidades. Disponível em: http://www.cidades.ibge.gov.br/. Acesso em 25 de Out. 2016.

Instituto Nacional de Pesquisas Espaciais (INPE). Catálogo de Imagens. Disponível em: http://www.dgi.inpe.br/CDSR/. Acesso em: 21 Set 2016.

LEPSCH, I. F. 19 lições de Pedologia. Oficina de Texto, São Paulo. 2001.

LIMA, A. H. S.; SILVA, P. E. D. \& MUSSE, N. S. 2012. A Situação Atual do Setor Mineral no Rio Grande do Norte e Suas Perspectivas de Futuro. In: VII Congresso Norte Nordeste de Pesquisa e Inovação, Palmas/ Tocantins, 2012. Anais. 06p.

MENEZES, P. T. L; ROIG, H. L; SILVA, G. B; MANE, M. A. PROSPECÇÃO MINERAL DO GRUPO PARANOÁ. REPROCESSAMENTO DE AEROGAMAESPECTROMÉTRICOS E INTERPRETAÇÃO INTEGRADA A IMAGENS TM LANDSAT 5. Revista Brasileira de Geofísica. Vol.24 no.3, São Paulo, 2006..

POUCHAIN, A. C. 2011. A Natureza Jurídica da Concessão de Lavra Mineral no Brasil. Universidade de Brasília, Monografia (graduação em Direito), 83p.

Serviço Geológico do Brasil (CPRM). 2005. Projeto Cadastro de Fonte de Abastecimento por Água Subterrânea Diagnóstico do Município de Caicó. Org. Mascarenhas et al. Recife: CPRM/PRODEEM. 
Sistemas de Informações Geográficas (SIGMINE). Web map. DNPM. Disponível em: http://sigmine.dnpm.gov.br/webmap/. Acesso em 21 Jul 2016.

Recebido em: 31/07/2017

Aceito para publicação em: 20/11/2017 